\title{
The astroclimate of Maidanak Observatory in Uzbekistan
}

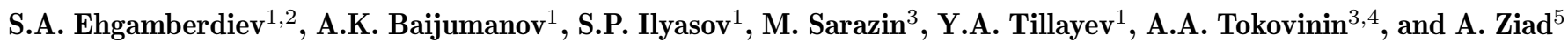 \\ 1 Ulugh Beg Astronomical Institute of the Uzbek Academy of Sciences, Astronomicheskaya 33, 700052 Tashkent, Uzbekistan \\ 2 Max-Plank-Institute fur Astrophysik, Karl-Schwarzschild-Str. 1, 85748 Garching, Germany \\ 3 European Southern Observatory, Karl-Schwarzschild-Str. 2, 85748 Garching, Germany \\ 4 Sternberg Astronomical Institute, Universitetsky Prosp. 13, 119899 Moscow, Russia \\ ${ }^{5}$ UMR 6525 Astrophysique, Université de Nice, Parc Valrose, 06108 Nice Cedex 2, France
}

Received April 28; accepted May 22, 2000

\begin{abstract}
The atmospheric turbulence and meteorology of the Maidanak Observatory in Uzbekistan are reviewed. Night time seeing was measured during the period August 1996 - November 1999 with the ESO Differential Image Motion Monitor. The median zenith seeing (FWHM) for the entire period of observations is $0.69^{\prime \prime}$ at $0.5 \mu \mathrm{m}$. A maximum clear sky season for Maidanak is July September, with about $90 \%$ of possible clear time and a median seeing of $0.69^{\prime \prime}$. The best monthly median seeing, $0.62^{\prime \prime}$, is observed in November. The winter maximum of clear time is usually observed in February (up to 50\%) with a FWHM of $0.77^{\prime \prime}$. During an additional site testing campaign (9 nights) organized in July 1998, the median wavefront outer scale $\mathcal{L}_{0}$ of $25.9 \mathrm{~m}$ and a median isoplanatic angle $\theta_{0}$ of $2.48^{\prime \prime}$ were measured with the Generalized Seeing Monitor developed at the University of Nice. The temporal evolution of the wavefront can be described by several layers moving at slow velocities with predominant direction from the West. This corresponds to a remarkably large atmospheric time constant. No correlation between wavefront velocity and the wind velocity at ground level was found. The good seeing, large isoplanatic angle and, especially, slow wind, place Maidanak Observatory among the best international astronomical sites for high angular resolution observations by interferometry and adaptive optics.
\end{abstract}

Key words: atmospheric effects — site testing

\section{Introduction}

Maidanak Observatory (longitude $66^{\circ} 56^{\prime}$ E, latitude $\left.38^{\circ} 41^{\prime} \mathrm{N}\right)$ is located at the south-east of the Republic

Send offprint requests to: S.A. Ehgamberdiev

Correspondence to: shuhrat@astrin.uzsci.net of Uzbekistan at a distance of about $120 \mathrm{~km}$ south of Samarkand. It lies on the spurs of the Pamir and Alai mountain system at $2600 \mathrm{~m}$ above sea level. The landscape of the observatory is mainly smooth and rock outcrops do not prevent from building over the whole 30 hectares of its territory. The vegetation of Mt. Maidanak is typical of high-mountain dry sub-tropics, bushes being prevailing. This summit was selected for astronomical observations in the late 60 -s as a result of a ten-year long site assessment campaign organized at the Ulugh Beg Astronomical Institute (UBAI) of the Uzbek Academy of Sciences by V.S. Shevchenko and his co-workers (Slutsky \& Khetselius 1970; Kardopolov et al. 1971; Shevchenko 1973). The crucial role of V.S. Shevchenko in the development of Maidanak Observatory must be stressed here.

In the early 70 -s, when the high-quality atmospheric conditions of Mt. Maidanak have been established, the summit was occupied by a Satellite Laser Ranging (SLR) station (Scott 1995). Nevertheless, UBAI astronomers were allowed to continue astronomical observations with two $60-\mathrm{cm}$ and one $48-\mathrm{cm}$ telescopes in the neighborhood of SLR station. Meanwhile the Moscow State University (MSU) and a few other Soviet Union institutions had erected their astronomical facilities at a neighboring summit situated $5 \mathrm{~km}$ to the west of Mt. Maidanak. So, there are actually two "Maidanak" summits - the Eastern, hosting SLR station and UBAI Observatory, and the Western, hosting all other observatories. By the early 90 -s about 10 telescopes as well as the corresponding observatory infrastructure (roads, buildings, mechanical shops, etc.) were equipping the Western summit. At present all astronomical facilities of both summits belong to UBAI and are operated in the framework of scientific agreements between UBAI and MSU, Ukrainian and Lithuanian Astronomical Institutions. Hereafter we call "Maidanak Observatory" the Western summit where all the site testing studies described below have been carried out. 


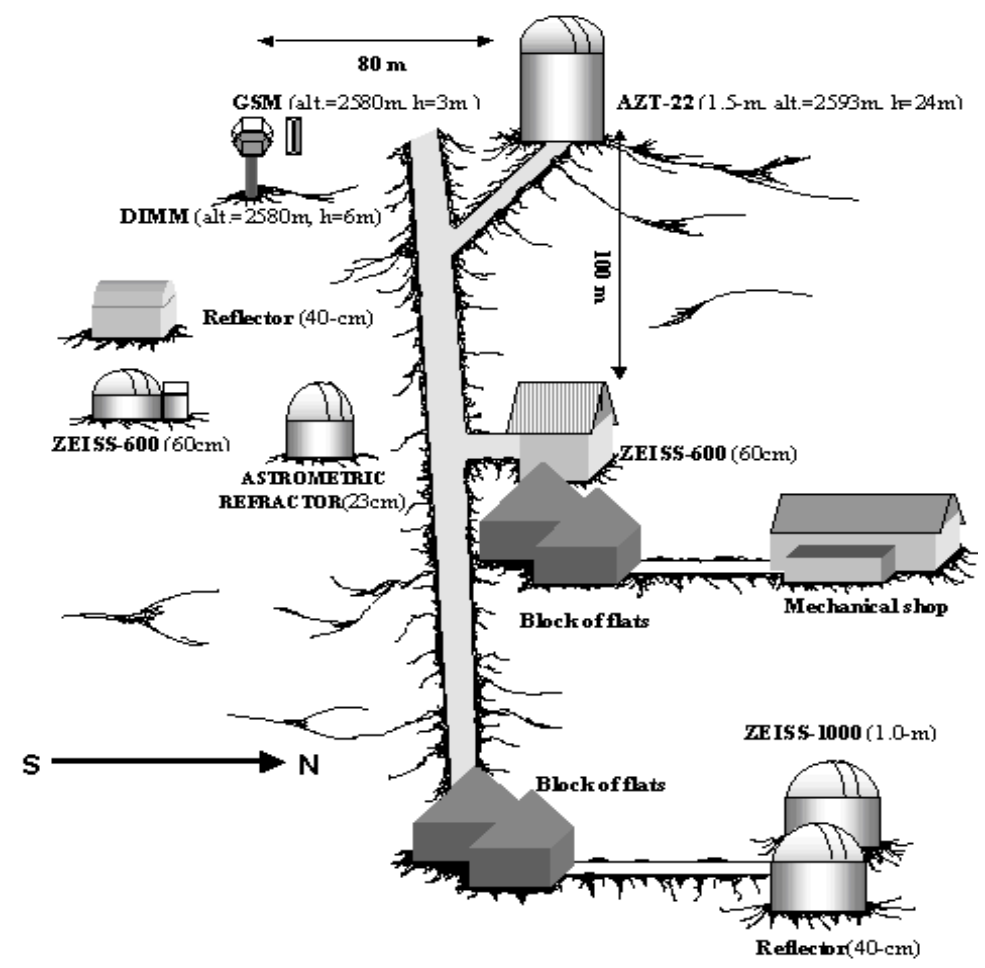

Fig. 1. Maidanak Observatory layout

The layout of Maidanak Observatory with the location of its telescopes is shown in Fig. 1.

The studies of the Maidanak astroclimate continued through the $80-\mathrm{s}$ and up to the beginning of the $90-\mathrm{s}$ in close cooperation between UBAI and MSU astronomers (Artamonov et al. 1987; Shcheglov \& Gur'yanov 1991). They provided a detailed meteorological information, such as the amount of clear time, temperature variations, wind speed, humidity and their seasonal distribution (Novikova 1970; Gladyshev \& Shirokova 1987). The measurements of extinction coefficient (Zheleznyakova 1984), sky background (Kardopolov \& Philip'ev 1979), as well as comprehensive optical and aerophysical studies (Gur'yanov et al. 1992) (hereafter Gur92) were carried out as well. Measurements of the seeing, a basic parameter of the atmosphere of an astronomical site, have been repeatedly conducted at Mt. Maidanak by several groups of researchers using different methods and instruments (Shevchenko 1973; Artamonov et al. 1987; Shcheglov \& Gur'yanov 1991; Ilyasov et al. 1992; Gur'yanov et al. 1992).

All these studies indicated that Mt. Maidanak was one of the most favorable sites worldwide for astronomical observations. Not only this fact, but also an exceptional geographical location at a mid-distance between the main international astronomical facilities of Chile, Hawaii and Canaries, make Mt. Maidanak very attractive for observational programs requiring continuous monitoring of astronomical objects. By now several observational programs using focal instrumentation received from advanced western institutions are already being carried out at Mt. Maidanak (Schulte-Ladbeck 1997; Herbst 1999; Nelsen 1999).

However, for many observational programs it was necessary to establish whether atmospheric conditions of Mt. Maidanak match the requirements of modern highresolution astronomical observation. In this paper we present the results of the statistical analysis of seeing measurements carried out at Mt. Maidanak with the ESO DIMM during the period from August 1996 up to October 1999 (Sect. 2). In Sect. 2 we present also a comparison of the results obtained in this work with seeing conditions at ESO Observatories of La Silla and Paranal in Chile and at Roque de los Muchachos Observatory (ORM) at La Palma.

When the high-quality atmospheric conditions of Mt. Maidanak have been established with a modern instrumentation, the next step was to measure other atmospheric parameters relevant to adaptive optics and interferometry, such as wavefront outer scale, isoplanatic angle, high altitude wind. In July 1998 during a site testing campaign, supported by INTAS ${ }^{1}$, all these parameters were measured with the Generalized Seeing Monitor (GSM) developed at the University of Nice (Martin et al. 1994). A brief description of the method used and results

\footnotetext{
1 International Association for the promotion of co-operation with scientists from the New Independent States of the former Soviet Union.
} 


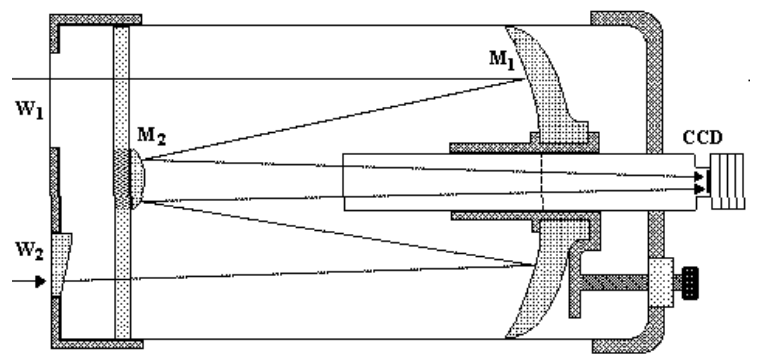

Fig. 2. Optical scheme of the Differential Image Motion Monitor (DIMM): M1, M2 are the parabolic primary and hyperbolic secondary mirrors, W1 and W2 are the entrance apertures. Two stellar images are separated on the detector by a wedge prism on W2

of the campaign, as well as cross-calibration of DIMM and GSM, are presented in Sect. 3. In Sect. 4 general meteorological information, estimation of wind speed and direction at different levels and correlation of seeing with ground wind speed are presented. In Sect. 5 the relative contribution of the different atmospheric layers to the seeing at Mt. Maidanak is discussed. The summary and final remarks are given in Sect. 6 .

\section{Seeing measurements with the DIMM}

A simplified and portable version of the standard ESO DIMM described in (Sarazin \& Roddier 1990) was used at Maidanak. It is based on a standard Celestron-11 telescope with a $279 \mathrm{~mm}$ primary mirror and a focal length of $2800 \mathrm{~mm}$ (Fig. 2). The pupil is covered by a mask with two circular $80 \mathrm{~mm}$ diameter apertures $200 \mathrm{~mm}$ apart. The mask is fixed above the Schmidt corrector plate. A prism with a wedge angle of $195^{\prime \prime}$ is added to one of the holes. The light detector is an ST5 CCD array with $10 \times 10 \mu \mathrm{m}$ square pixels. The instrument produces two stellar images on the detector, 121 pixels apart at the nominal focus (the plate scale is $0.73^{\prime \prime}$ per pixel).

Routine measurements were carried out on top of a $6 \mathrm{~m}$ high pillar placed on the slope of the main summit at the distance $80 \mathrm{~m}$ south of the main $1.5 \mathrm{~m}$ telescope of the Maidanak Observatory (Fig. 1). Standard DIMM operation consists in pointing a star brighter than +2.5 magnitude, within $30^{\circ}$ from zenith $( \pm 2 \mathrm{~h}$ hour angle) that allows to reduce observations to the zenith with a sufficient accuracy. Equivalent long exposure seeing is computed from the rms differential image motion in the direction of the baseline (longitudinal) and perpendicular to the baseline (transverse) estimated each 4 minutes from interlaced series of 40 individual frames of $\tau_{1}=10$ and $\tau_{2}=20 \mathrm{~ms}$. This low data rate is due to the limited speed of transfer in the serial link between the detector controller and the computer. As a consequence, the statistical error on the

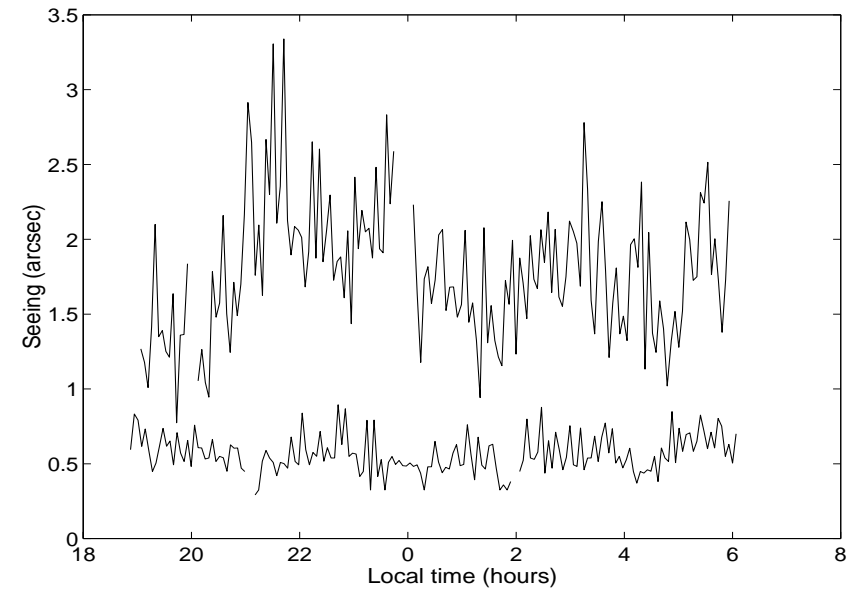

Fig. 3. Maidanak seeing measured by DIMM in arcseconds at zenith and $0.5 \mu \mathrm{m}$ : examples of a good and a bad nights

image motion variance estimate is the dominating noise term, of the order of $\pm 1 / \sqrt{39}$ or $\pm 16 \%$. This random error is fortunately averaged out in long term statistics, but well apparent on individual measurements as shown in Fig. 3, presenting samples of seeing records for a good and bad nights, respectively.

The finite duration of exposure time was taken into account by using the relation (Sarazin 1997a):

$\sigma_{\tau=0}^{2}=\sigma_{\tau_{1}}^{2} \frac{\sigma_{\tau_{1}}^{2}}{\sigma_{\tau_{2}}^{2}}$.

Because of the low wind speed, the correction factor $\sigma_{\tau_{1}}^{2} / \sigma_{\tau_{2}}^{2}$ had typically small values of $1.1-1.4$ over the entire period of observations at Maidanak.

The full series of seeing measurements obtained at Mt. Maidanak from August 1996 to November 1999 are shown in Fig. 4 and summarized in Table 1. The total number of nights covered over the entire 1180 days period was 483 , with an average duration of 8 hours. The corresponding histogram of the seeing estimates at Maidanak, including all DIMM measurements from 1996 to 1999, is shown in Fig. 5.

The median and mean values of the seeing for the entire period of observation were $0.69^{\prime \prime}$ and $0.76^{\prime \prime}$, respectively. The seasonal trends are shown in Table 2 where monthly medians are computed after merging different years. The winter period, which also provides a smaller percentage of clear nights, has the most turbulent atmosphere.

A comparison of the present results with the previous estimations of seeing at Mt. Maidanak shows that past data sets are unfortunately not entirely consistent. However, the reasons for the discrepancy with some Polar star motion measurements has been understood and these data can probably be used after re-calibration to analyze the long term stability of the site (Ilyasov et al. 1999).

Four international astronomical observatories (including Maidanak) have systematic seeing measurements 
Table 1. Monthly statistics of the seeing at Maidanak for the period August 1996 to October 1999

\begin{tabular}{|c|c|c|c|c|c|}
\hline Date & $N$ & $N$ & \multicolumn{3}{|c|}{ Seeing (arcsec) } \\
& nights & data & $25 \%$ & $50 \%$ & $75 \%$ \\
\hline 1996 & & & & & \\
\hline Aug. & 21 & 1802 & 0.52 & 0.67 & 0.85 \\
Sep. & 23 & 2094 & 0.55 & 0.71 & 0.90 \\
Oct. & 24 & 2589 & 0.52 & 0.72 & 0.96 \\
Nov. & 20 & 2104 & 0.48 & 0.60 & 0.75 \\
Dec. & 17 & 1953 & 0.55 & 0.69 & 0.89 \\
\hline 1997 & & & & & \\
\hline Jan. & 14 & 1320 & 0.62 & 0.98 & 1.31 \\
Feb. & 18 & 2388 & 0.58 & 0.74 & 0.94 \\
Apr. & 9 & 836 & 0.63 & 0.80 & 1.04 \\
Aug. & 16 & 1827 & 0.53 & 0.68 & 0.87 \\
Sep. & 19 & 3877 & 0.54 & 0.68 & 0.84 \\
\hline 1998 & & & & & \\
\hline Jun. & 4 & 150 & 0.59 & 0.81 & 1.02 \\
Jul. & 30 & 2633 & 0.53 & 0.68 & 0.89 \\
Aug. & 21 & 2301 & 0.57 & 0.72 & 0.92 \\
Sep. & 28 & 3213 & 0.53 & 0.67 & 0.84 \\
Oct. & 27 & 3753 & 0.56 & 0.72 & 0.93 \\
Nov. & 19 & 2672 & 0.50 & 0.63 & 0.79 \\
Dec. & 15 & 1492 & 0.59 & 0.73 & 0.93 \\
\hline 1999 & & & & & \\
\hline Jan. & 15 & 1299 & 0.61 & 0.78 & 0.96 \\
Feb. & 6 & 665 & 0.72 & 0.90 & 1.12 \\
Mar. & 7 & 684 & 0.52 & 0.64 & 0.79 \\
Apr. & 11 & 1057 & 0.61 & 0.79 & 1.00 \\
May & 21 & 2015 & 0.53 & 0.66 & 0.86 \\
Jun. & 25 & 2083 & 0.55 & 0.69 & 0.90 \\
Jul. & 24 & 1785 & 0.56 & 0.70 & 0.87 \\
Aug. & 10 & 1075 & 0.59 & 0.75 & 0.96 \\
Sep. & 19 & 2602 & 0.54 & 0.69 & 0.90 \\
Oct. & 20 & 2795 & 0.50 & 0.62 & 0.77 \\
\hline All & 483 & 53064 & 0.55 & 0.69 & 0.90 \\
\hline
\end{tabular}

Table 2. Seasonal statistics of the median seeing at Maidanak for the period August 1996 to October 1999 sorted by month

\begin{tabular}{|c|c|c|c|}
\hline Month & $N$ nights & $N$ data & $\beta_{\text {atm }}$, arcsec \\
\hline Jan. & 29 & 2619 & 0.84 \\
\hline Feb. & 24 & 3053 & 0.77 \\
\hline Mar. & 7 & 684 & 0.64 \\
\hline Apr. & 20 & 1893 & 0.80 \\
\hline May & 21 & 2015 & 0.66 \\
\hline Jun. & 29 & 2233 & 0.70 \\
\hline Jul. & 54 & 4418 & 0.69 \\
\hline Aug. & 68 & 7005 & 0.70 \\
\hline Sep. & 89 & 11786 & 0.68 \\
\hline Oct. & 71 & 9137 & 0.68 \\
\hline Nov. & 39 & 4776 & 0.62 \\
\hline Dec. & 32 & 3445 & 0.71 \\
\hline All & 483 & 53064 & 0.69 \\
\hline
\end{tabular}
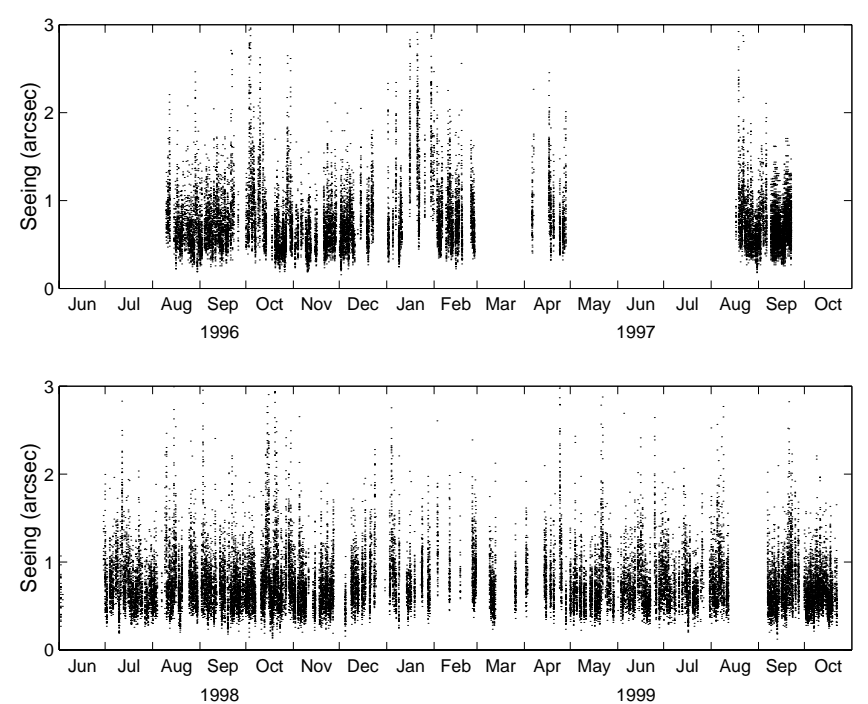

Fig. 4. Whole span of seeing measurements obtained at Maidanak with the DIMM for the period August 1996 to October 1999

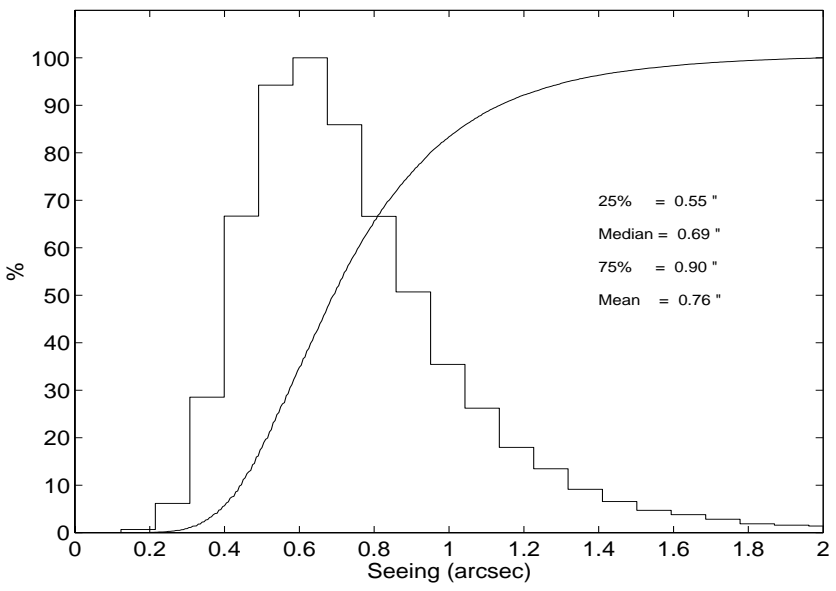

Fig. 5. Statistical distribution of Maidanak seeing from the measurements shown in Fig. 4

taken with cross-calibrated DIMMs. In Fig. 6 we present the cumulative distributions of seeing at Paranal and La Silla measured over the period [1989-1995] (Sarazin 2000), Roque de los Muchachos Observatory (ORM) at La Palma over the period [1994-1998] (Munoz-Tuñon et al. 1999; Wilson 2000) and at Maidanak [1996-1999]. The median value of the seeing at ORM measured by ING and DA/IAC DIMMs is $0.69^{\prime \prime}$, whereas at La Silla and Paranal Observatories it is $0.87^{\prime \prime}$ and $0.66^{\prime \prime}$, respectively. We can conclude that the atmosphere above Mt. Maidanak is on a par with the conditions at the world's leading observatories. Regarding seasonal variations, Fig. 7 shows that Paranal and Maidanak, belonging to different hemispheres, are slightly anti-correlated. However, the seasonal seeing modulation is only about $\pm 10 \%$. 


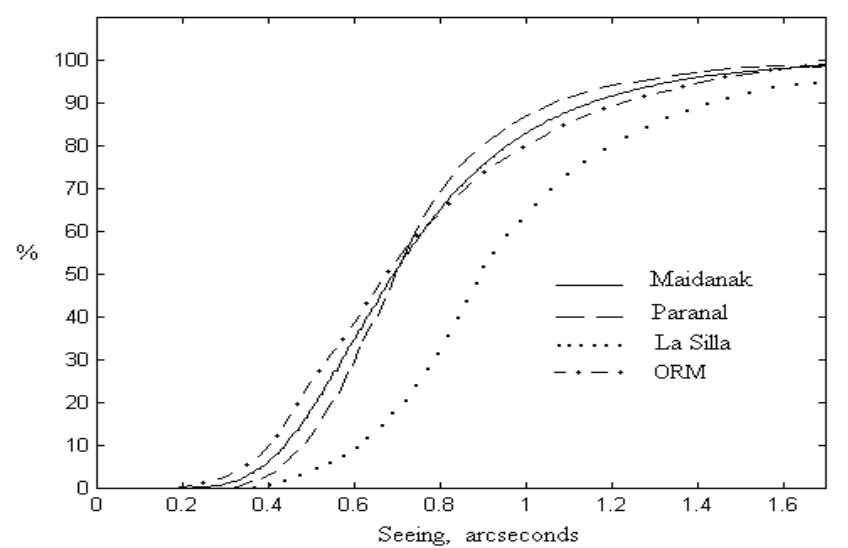

Fig. 6. Statistical distribution of the seeing at Maidanak [19961999]: comparison with ESO observatories of La Silla and Paranal [1989-1995] and Roque de los Muchachos Observatory at La Palma [1996-1999]

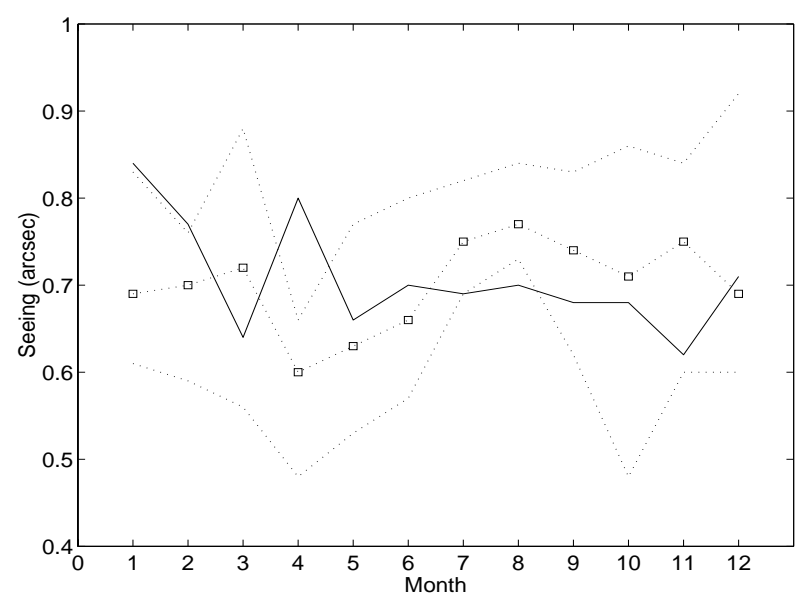

Fig. 7. Monthly median seeing at Maidanak in [1996-1999] (full line) and at Paranal (squares on dotted line) with the minimum and maximum monthly averages (dotted lines) during the period [1993-1999]

\section{Generalized seeing monitor}

\subsection{Instrument layout}

In view of monitoring additional atmospheric parameters - the wavefront outer scale $\mathcal{L}_{0}$, the wavefront coherence time $\tau_{0}$ and the isoplanatic angle $\theta_{0}$, - which are of particular importance to adaptive optics and interferometry, a specific campaign was organized in July 1998. All these parameters were measured with GSM developed at Nice University in collaboration with MSU (Martin et al. 1994). The GSM was first extensively used in 1997 during the La Silla campaign in Chile (Martin et al. 1998; Tokovinin et al. 1998), then in 1998 at the Gemini site of Cerro Pachon (Vernin et al. 2000) and at the VLT site of Paranal (Martin et al. 2000).
GSM consists of four identical units for angle of arrival (AA) measurements. Each unit has a $10 \mathrm{~cm}$ Maksutov telescope and a detection module to measure the stellar image position using a grating in the focal plane together with fast $(200 \mathrm{~Hz})$ modulation. The light transmitted by the grating is detected by a photo-multiplier in pulse counting mode. All four units work synchronously and provide the angle of arrival data with $5 \mathrm{~ms}$ temporal resolution. The units were equipped with simple but rigid alt-azimuth supports pointing the Polar star. The field of view of $13.7^{\prime}$ permits observations during $30 \mathrm{~min}$ without re-pointing. Units 1 and 2 had a common support for DIMM-like seeing measurement in differential mode (baseline $25 \mathrm{~cm}$ ). GSM was installed on a $3 \mathrm{~m}$ high rectangular arch-shape concrete pier, the optical apertures were $3.4 \mathrm{~m}$ above ground level. Unit 3 was located on the same pier to the east, $0.8 \mathrm{~m}$ from the center of the differential unit, and unit 4 was located $1.5 \mathrm{~m}$ below (1.9 $\mathrm{m}$ above ground). All units measure AA fluctuations in the horizontal direction. Thus the baselines formed by units $1-2,1-3$, $2-3$ are longitudinal, baselines $1-4$ and $2-4$ are approximately transverse, and baseline $3-4$ is oblique. Such a geometrical arrangement was chosen to explore the $2 \mathrm{D}$ structure of the wavefront and to determine its effective speed. Baseline lengths are optimized for maximum sensitivity to the outer scale effects for the expected decametric outer scale values. In the previous GSM missions all GSM units were located in a horizontal plane, but our present " $3 \mathrm{D}$ " arrangement results in the same unit configuration as projected onto the wavefront.

Data acquisition was typically $2 \mathrm{~min}$ long, repeated every $4 \mathrm{~min}$. Data were processed immediately after acquisition. Processing includes calculation of the AA variance in each unit and AA covariances which, after suitable normalization, were compared to the pre-calculated table to find the wavefront outer scale $\mathcal{L}_{0}$. The six values of $\mathcal{L}_{0}$, corresponding to the 6 baselines of GSM, are found, and their median is taken as final result. The $\mathcal{L}_{0}$ provided by GSM is an outer scale parameter in the Von Kármán turbulence model, its relation to outer scale in some other models is studied in (Ziad et al. 2000).

Seeing is calculated from the differential AA variance in units 1 and 2, like in DIMM. Small corrections for photon and scintillation noise are made. Correction for finite exposure time is also performed by calculating the AA variance with $5 \mathrm{~ms}$ and $10 \mathrm{~ms}$ temporal resolution and extrapolating to the $0 \mathrm{~ms}$ exposure time. This latter correction was typically about $10 \%$, because seeing at Maidanak is rather slow. Additional estimation of seeing comes from the AA variance in each unit, with a nonnegligible correction for finite outer scale. A good match between differential and absolute seeing estimates is a sign that the support vibration was not important, which is a necessary condition for correct GSM operation. Also, the difference between AA variances measured by the units 3 and 4 was used to estimate the importance of ground layer 
Table 3. GSM data summary: number of hours $T$ covered each night and the number of data points $N$. Median seeing $\beta$, median isoplanatic angle $\theta_{0}$ at zenith and $0.5 \mu \mathrm{m}$ and median $\mathcal{L}_{0}$ for each night and for the whole data set

\begin{tabular}{cccccc}
\hline $\begin{array}{c}\text { Date } \\
\text { July 1998 }\end{array}$ & $\begin{array}{c}T \\
\mathrm{~h}\end{array}$ & $N$ & $\begin{array}{c}\beta_{\text {med }} \\
\text { arcsec. }\end{array}$ & $\begin{array}{c}\theta_{\text {0med }} \\
\text { arcsec. }\end{array}$ & $\begin{array}{c}\mathcal{L}_{\text {0med }} \\
\mathrm{m}\end{array}$ \\
\hline 16 & 4.4 & 69 & 0.96 & 2.02 & 32.6 \\
17 & 7.0 & 100 & 0.58 & 2.49 & 27.2 \\
18 & 4.7 & 99 & 0.74 & 2.24 & 22.6 \\
19 & 6.2 & 96 & 0.52 & 3.49 & 26.2 \\
20 & 7.4 & 104 & 0.53 & 3.43 & 23.2 \\
21 & 7.3 & 101 & 0.63 & 2.48 & 27.3 \\
22 & 6.9 & 99 & 0.87 & 2.41 & 34.6 \\
23 & 7.4 & 103 & 0.74 & 2.52 & 22.8 \\
24 & 7.6 & 79 & 0.79 & 1.79 & 22.7 \\
25 & 0.5 & 7 & 1.21 & 1.14 & 25.0 \\
\hline Total & 58.9 & 850 & 0.68 & 2.48 & 25.9 \\
\hline
\end{tabular}

turbulence, because these units were installed at different heights above ground (3.4 and $1.9 \mathrm{~m}$, respectively).

The isoplanatic angle was calculated from the scintillation index, as explained in (Conan et al. 1999; Ziad et al. 2000). Our time resolution of $5 \mathrm{~ms}$ is not sufficient for unbiased measurement of scintillation, but we corrected for time averaging in the same manner as for the AA.

\subsection{Summary of GSM results}

The 10 days measurement campaign took place in July, a month representative of the best observing period at Maidanak which extends from mid-summer to early autumn. The probability of good weather for this period is very high. Indeed, our mission started on July 16, in the conditions of stable weather with clear sky and good seeing, which persisted during the first 8 nights (July 16-23). Then, on July 24, 2 hours were lost due to cloud passages. On the night of July 25 only few GSM observations were made, because of the fog and associated humidity (the previous day was rainy). This last night is considered as lost due to weather, although the scintillation and DIMM measurements were taken as planned in its second half. In summary, we had 8 clear and 2 partially clear nights out of 10 . Table 3 summarizes the measurements collected during this campaign.

\subsection{Outer scale and isoplanatic angle}

The measured $\mathcal{L}_{0}$ values, average $31.5 \mathrm{~m}$ and median $25.9 \mathrm{~m}$, are typical of other GSM missions. The median isoplanatic angle $\theta_{0}$ is $2.48^{\prime \prime}$, mean is $2.57^{\prime \prime}$. The histograms of the outer scale and isoplanatic angle during the 9 nights of the mission are given in Fig. 8. All atmospheric parameters are reduced to observations at zenith at the wavelength of $500 \mathrm{~nm}$.
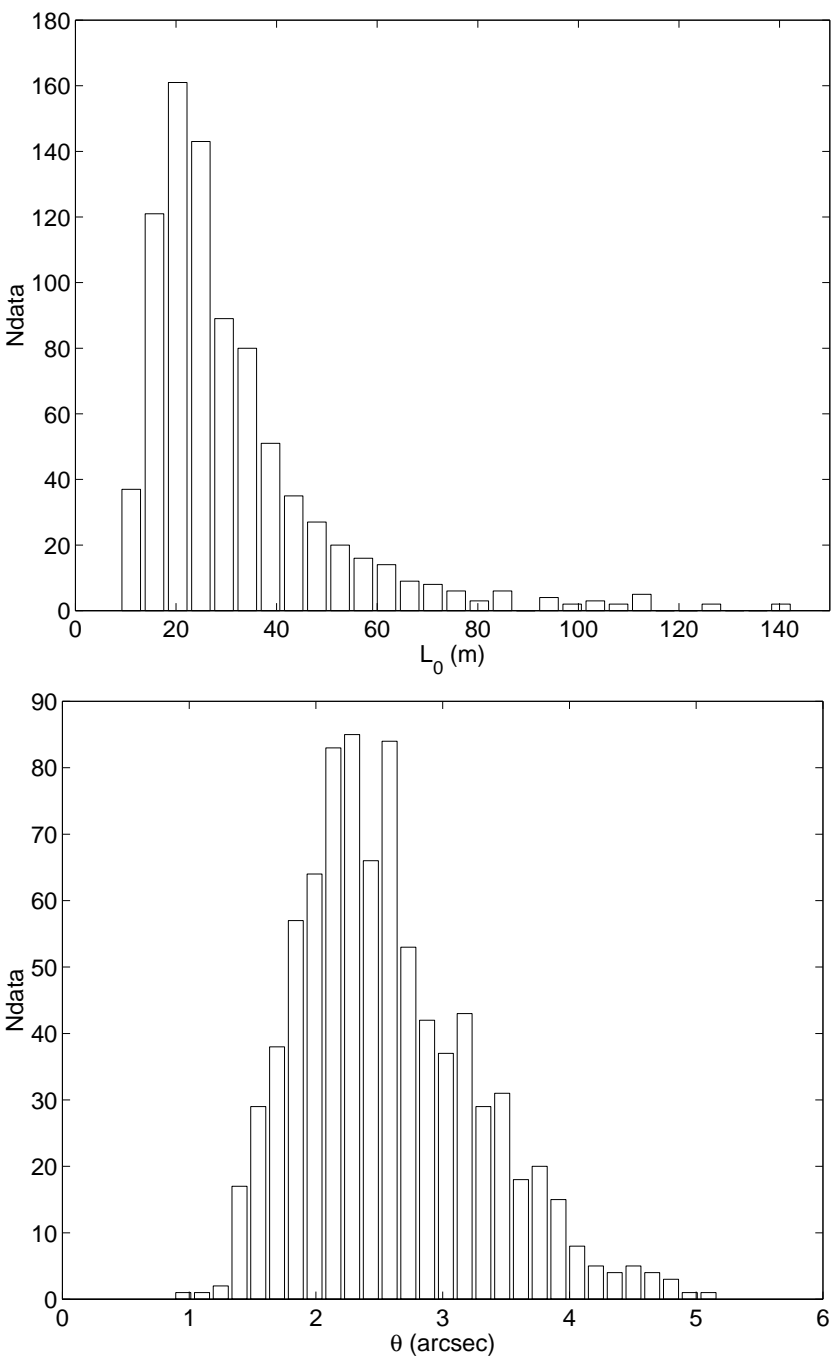

Fig. 8. The histograms of the outer scale (top) and isoplanatic angle (bottom) estimations with GSM at Mt. Maidanak

\subsection{Wavefront velocities and time constant}

The temporal characteristics (velocity and direction) of the predominant turbulent layers of the atmosphere were determined from the analysis of AA temporal crosscorrelation functions at all GSM baselines (Conan et al. 1999). The eddies carried by the winds in the layers produce several peaks in the cross-correlations. With the hypothesis of the frozen-flow, the positions of the peaks depend on the baseline and on the projection of the wind on the baseline as shown by Avila et al. (1997). Practically, the positions of the highest peak are measured on the six cross-correlations. To reconstruct the wind vector, two non-parallel baselines are necessary. With the six baselines, 11 couples of equations lead to the 11 measurements of the wind vector. The final value is the median of these. This procedure turned out to be rather robust, and practically it gives the velocity of dominant turbulent layers. When several layers are present (which was almost always 


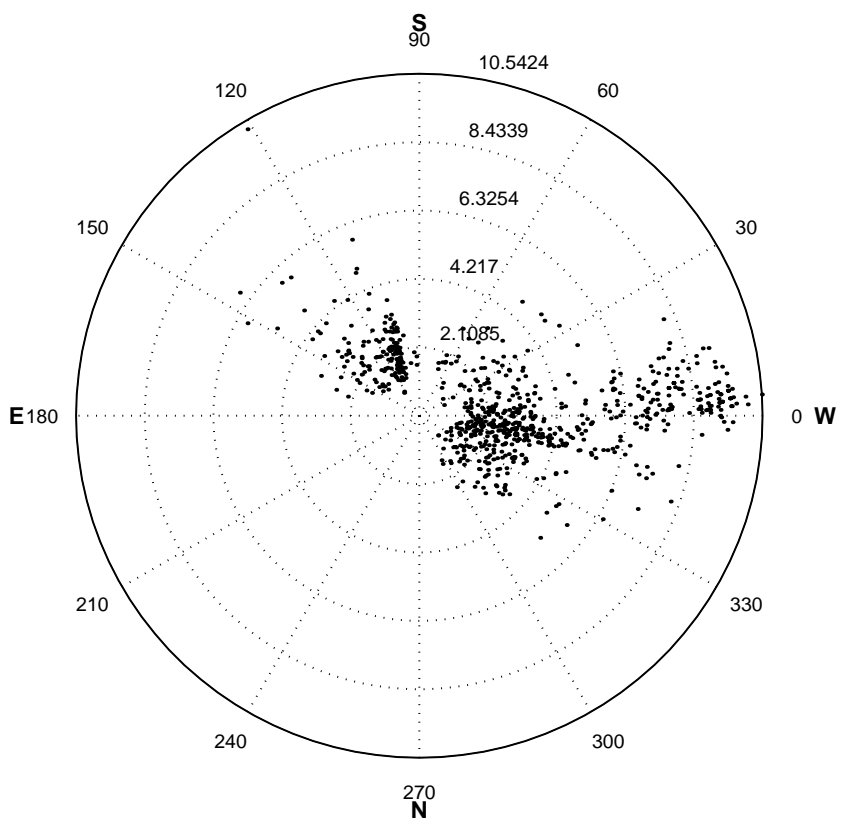

Fig. 9. Direction and velocity of the wavefront for the whole GSM mission data set, plotted as a polar diagram. The western wind of low to moderate velocity was predominant, with south-east winds being less frequent

the case), our wavefront velocity vectors often fluctuate between the values typical for each layer, because of turbulence intermittency. So, the plots of wind speed and direction against time usually reveal the multi-layer structure quite well.

The overall distribution of the direction and velocity of wavefront is given in Fig. 9. A predominant western direction is apparent. It corresponds to the planetary circulation in the free atmosphere. Very low wavefront speed is obtained: it was less than $4 \mathrm{~m} / \mathrm{s}$ most of the time, and never exceeded $10 \mathrm{~m} / \mathrm{s}$. Although still subject to theoretical interpretation, these measurements can be used to estimate the temporal coherence of the atmosphere: taking $V=4 \mathrm{~m} / \mathrm{s}$ as representative, we obtain the atmospheric time constant $\tau=0.31 r_{0} / V=12 \mathrm{~ms}$, that is 2 to 4 times longer than at other main observatories (Sarazin 1995; Vernin et al. 2000). Direct interferometric measurements over 19 nights at Maidanak also yielded a time constant of $12 \mathrm{~ms}$ (Tokovinin 1980).

\subsection{Cross-calibration of DIMM and GSM}

One of the important results obtained during the July 1998 campaign was cross-calibration of Maidanak DIMM with the GSM. As can be seen from Fig. 10, a good mutual agreement of GSM and DIMM seeing estimates exists most of the time. DIMM measurements show a larger scatter, explained by the small number of frames available for computing the variance of the image motion.
Table 4. Monthly average fraction of clear nights at Maidanak in [1979-1985] (Gladyshev \& Shirokova 1987) and of photometric nights at La Silla and Paranal during the period [1983-1997] (Sarazin 1997b)

\begin{tabular}{|c|c|c|c|}
\hline Month & Maidanak & La Silla & Paranal \\
\hline January & 31 & 73 & 77 \\
February & 54 & 79 & 84 \\
March & 42 & 76 & 83 \\
April & 47 & 63 & 79 \\
May & 44 & 40 & 70 \\
June & 79 & 34 & 70 \\
July & 92 & 42 & 75 \\
August & 81 & 48 & 73 \\
September & 84 & 54 & 85 \\
October & 55 & 57 & 83 \\
November & 42 & 67 & 84 \\
December & 46 & 69 & 77 \\
\hline Total & 58 & 59 & 78 \\
\hline
\end{tabular}

The $25 \%, 50 \%$ and $75 \%$ levels of the cumulative seeing distribution as measured during all 9 nights with GSM are $0.56^{\prime \prime}, 0.68^{\prime \prime}$ and $0.84^{\prime \prime}$. The corresponding values for DIMM are $0.52^{\prime \prime}, 0.64^{\prime \prime}$ and $0.82^{\prime \prime}$. DIMM observed stars near zenith while GSM observed Polaris, and hence both instruments sampled different volumes of the atmosphere. Correction of the GSM seeing to zenith was however not too large, $\left(\sec 51^{\circ}\right)^{0.6}=1.32$.

\section{Meteorology of the Maidanak Observatory}

\subsection{Clear time}

The number of clear night time hours is of course a major parameter defining the quality of a stellar observatory. The first serious work comparing different regions of Central Asia was carried out by Novikova (1970). It was shown that Mt. Maidanak could provide about 2000 clear night time hours per year. This was supplemented by Gladyshev \& Shirokova (1987) with an analysis based on clear time records in intervals from mid1978 to mid-85, and also including the $1 \mathrm{~m}$ telescope measurements in 1981-1983. The statistics compiled by Gladyshev \& Shirokova (1987) are compared in Table 4 to similar work made at the ESO observatories of La Silla and Paranal (Sarazin 1997b). The definitions of photometric time slightly differ in the two studies. To keep this in mind, we use the term "clear time" (the cloudness is not more than 25\%) for Maidanak and reserve "photometric time" (no clouds above 18 degree from horizon during at least 6 hours in a row) for ESO observatories.

As shown in Fig. 11, seasonal trends are stronger at Maidanak, with excellent summer months as was noted by previous authors. We note that with $58 \%$ of photometric nights, Maidanak is similar to the ESO observatory of La Silla in Chile. 

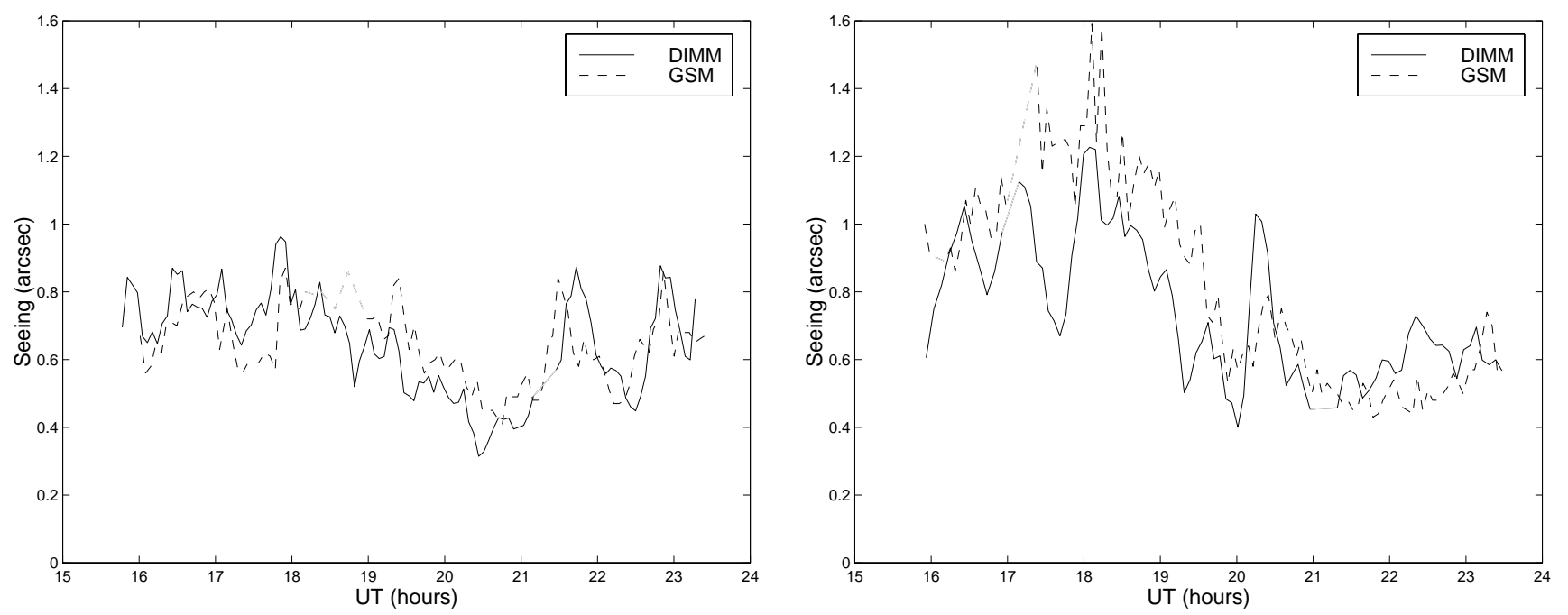

Fig. 10. Seeing measured by DIMM (solid line) and by GSM (dashed line) on the night of July 21-22 with the best seeing (left) and on July 23-24 with average seeing (right), when for the first 2 hours GSM units 1 and 2 were installed on the DIMM pier. DIMM data are smoothed by a running mean

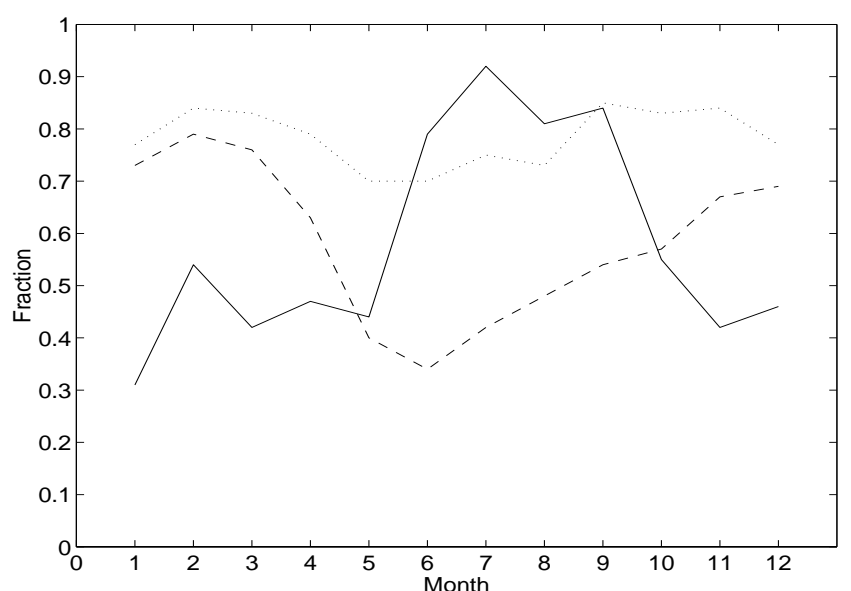

Fig. 11. Monthly average fraction of clear nights at Maidanak in [1979-1985] (full line) compared to the statistics of photometric nights at La Silla (dashed line) and Paranal (dotted line) during the period [1983-1997]. The yearly percentages for each site are respectively 58,59 and $78 \%$ of available dark time. Data are from Table 4

\subsection{Temperature}

In summer the average night temperature at Maidanak is $+13 \mathrm{C}$. In winter it can sometimes fall down to $-15 \mathrm{C}$. The night time temperature amplitude is about $5 \mathrm{C}$. Winter storms are possible, during which the wind velocity remains moderate $(15 \mathrm{~m} / \mathrm{s})$. For this study the wind velocity and air temperature have been measured every hour manually. The measurements were taken at $5 \mathrm{~m}$ above ground on the DIMM tower and are representative of the operating conditions of Maidanak astronomical facilities. The results for the whole campaign are presented in Table 5, stacked into one single year.

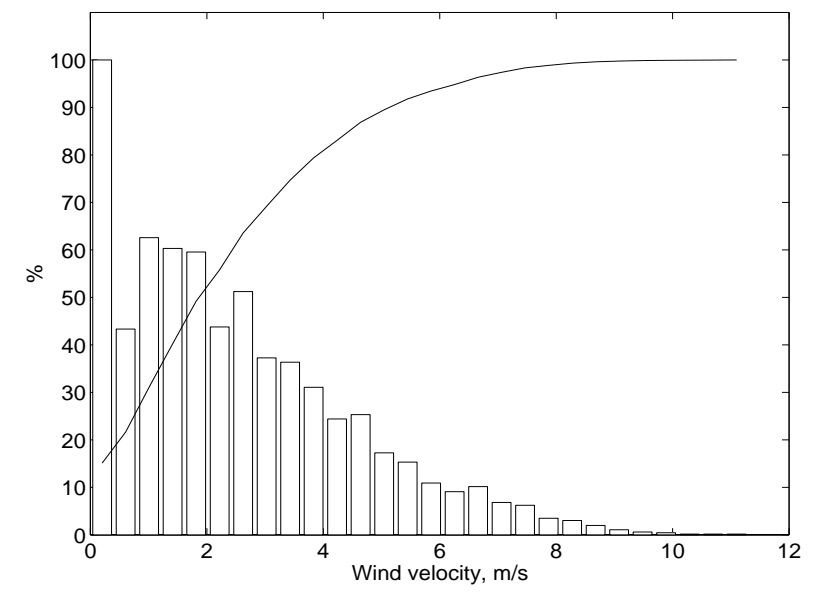

Fig. 12. Statistical distribution of the wind speed at Mt. Maidanak measured at $5 \mathrm{~m}$ above ground

\subsection{Ground level wind}

The wind speed was measured at the DIMM tower using a Fuss wind speedometer. The statistics presented in Fig. 12 corresponds to more than 3500 individual measurements. An overall average wind speed of $2.1 \mathrm{~m} / \mathrm{s}$ was measured with a prevailing South-East wind direction (Fig. 13). This is a remarkably low value for a mountain site. All these data are in agreement with previous observations (Gladyshev \& Shirokova 1987).

\subsection{Relation of seeing with ground wind speed}

The seeing shows some weak correlation with wind speed at the DIMM location (13 $\mathrm{m}$ below the mountain ridge). A fitting relation is proposed in Fig. 14 for the available 
Table 5. Seasonal statistics of wind velocity $(\mathrm{m} / \mathrm{s})$ and temperature (Celsius) measured once per hour at $5 \mathrm{~m}$ above ground during DIMM seeing measurements at Maidanak for the period August 1996 to October 1999 reorganized on a 12 months basis

\begin{tabular}{|c|c|c|c|c|c|c|c|c|}
\hline & \multicolumn{3}{|c|}{ Wind, m/s } & \multicolumn{4}{c|}{ Temperature, Celsius } \\
Month & NData & Median & Max & Mean & Median & Min & Max & Mean \\
\hline 01 & 202 & 2.85 & 9.70 & 3.22 & -7.20 & -14.20 & 1.40 & -6.19 \\
\hline 02 & 239 & 1.00 & 8.00 & 1.96 & -6.80 & -15.40 & -1.00 & -7.01 \\
\hline 03 & 58 & 2.00 & 9.30 & 2.44 & -4.40 & -9.40 & -2.00 & -4.69 \\
\hline 04 & 99 & 3.75 & 8.50 & 3.55 & 2.70 & -4.00 & 6.20 & 2.00 \\
\hline 05 & 102 & 2.30 & 4.90 & 2.41 & 8.00 & 5.00 & 13.40 & 8.61 \\
\hline 06 & 206 & 3.20 & 9.00 & 3.33 & 11.60 & 5.80 & 17.20 & 11.74 \\
\hline 07 & 311 & 2.00 & 8.50 & 2.27 & 14.20 & 7.20 & 24.50 & 14.32 \\
\hline 08 & 409 & 2.00 & 10.70 & 2.26 & 14.20 & 4.40 & 19.50 & 13.56 \\
\hline 09 & 629 & 2.20 & 8.60 & 2.50 & 11.20 & 1.20 & 17.60 & 11.62 \\
\hline 10 & 691 & 2.00 & 10.00 & 2.27 & 5.20 & -4.80 & 12.50 & 4.99 \\
\hline 11 & 356 & 1.50 & 9.00 & 1.87 & 0.80 & -7.80 & 9.40 & 0.61 \\
\hline 12 & 142 & 0.50 & 6.80 & 1.16 & -6.65 & -13.60 & 2.00 & -6.43 \\
\hline
\end{tabular}

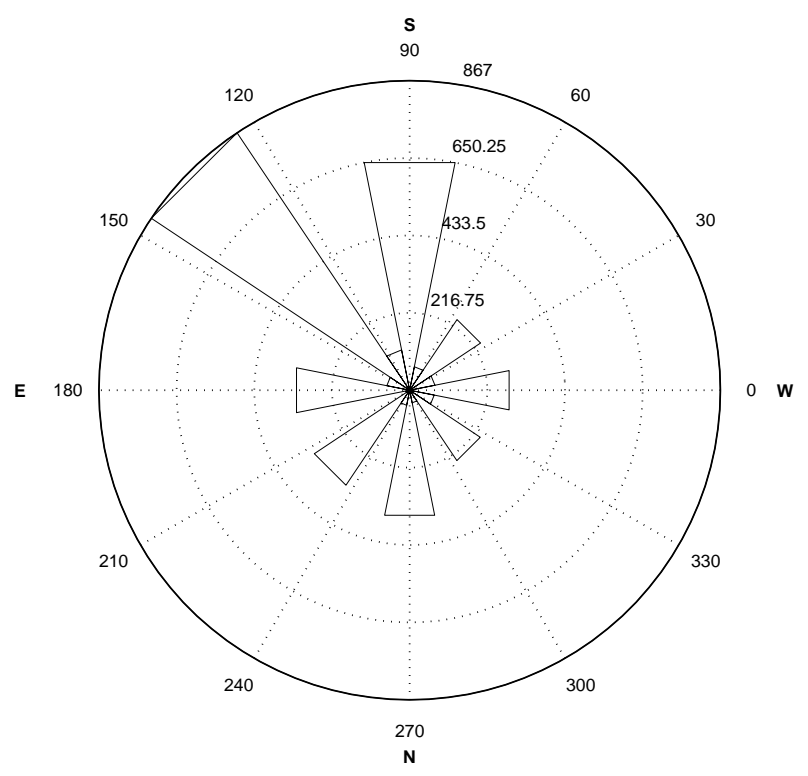

Fig. 13. Maidanak windrose measured at $5 \mathrm{~m}$ above ground

data set, showing that the best seeing conditions occur for wind speed lower than $5 \mathrm{~m} / \mathrm{s}$, i.e. in $90 \%$ of the cases.

\subsection{Wind profile in the boundary layer}

In Fig. 15 the average vertical profile of the wind speed obtained by balloon observations at Mt. Maidanak in 1990 is shown, as measured by E.I. Sofiev et al. and given in Gur92. The wind speed at ground level is in good agreement with the median value of $2.1 \mathrm{~m} / \mathrm{s}$ obtained from our data. The wavefront speed range $4-10 \mathrm{~m} / \mathrm{s}$, determined from GSM data, does not contradict this profile if we assume that the predominant turbulent layers are located in the wind shear zones.

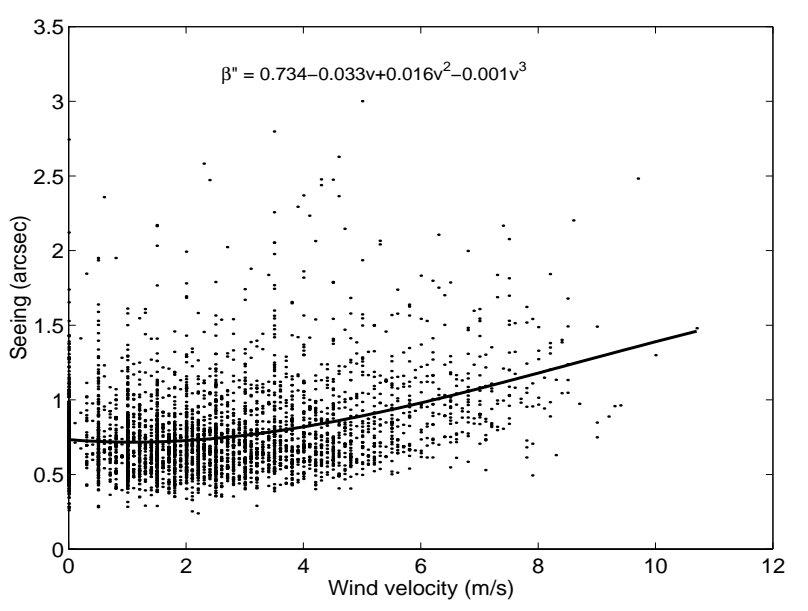

Fig. 14. Dependence of the seeing on ground-level wind speed at Mt. Maidanak

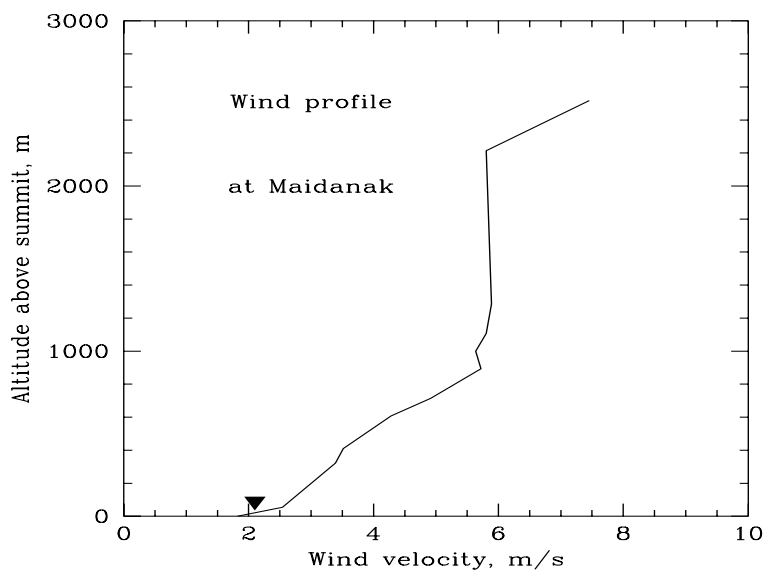

Fig. 15. Vertical profile of the average wind velocity obtained by balloon observations as given in Gur92. Our data are indicated by the triangle 


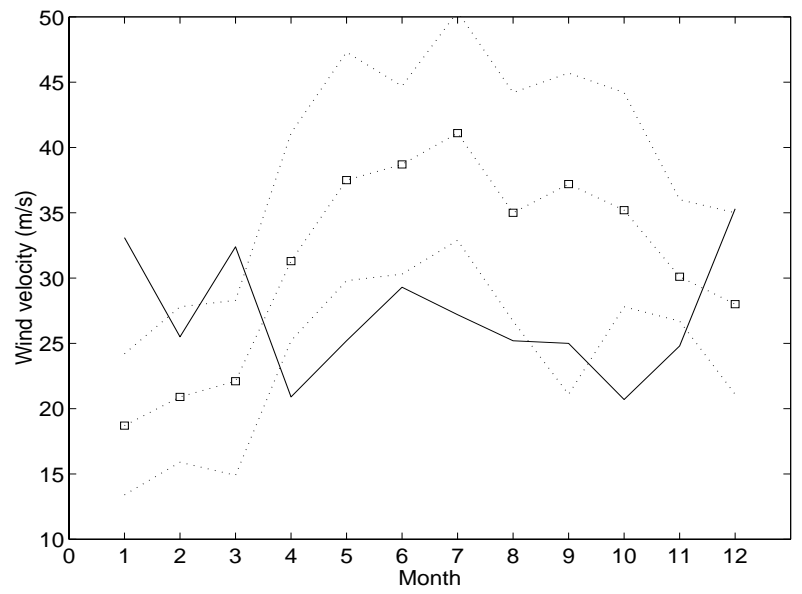

Fig. 16. Wind velocity at $200 \mathrm{mB}$ : monthly average velocity at Maidanak in [1991] (full line) and at Paranal (squares on dotted line) with the minimum and maximum monthly averages (dotted lines) during the period [1987-1999]

\subsection{High-altitude wind speed}

In order to estimate the wind speed at higher level we used the High Altitude Data provided by the Uzbek Meteorological Center. The tropopause level (200 mB pressure, or $12 \mathrm{~km}$ above sea) wind data were obtained only during the year 1991 at three meteorological stations in Uzbekistan: Kokand (400 km East of Mt. Maidanak), Tashkent (350 km North-East) and Termez (160 km South). Unfortunately, there are no high altitude measurements in the region of Mt. Maidanak, but the wind statistics of the three stations is very similar with prevailing western wind. We interpolated wind velocity and wind direction above Mt. Maidanak using weights proportional to the inverse of the distance of each station to Maidanak. We have calculated a median value of tropopause wind velocity of $26.8 \mathrm{~m} / \mathrm{s}$ and with predominantly western direction. Same direction with a lower speed was measured by GSM, as described in Sect. 3. It is thus the same planetary wind which extends down to $2.5 \mathrm{~km}$ above the site, with the bulk of the turbulence at this level.

For comparison, we give in Fig. 16 the seasonal variation of the average $200 \mathrm{mB}$ wind speed at Paranal (Sarazin 2000). Although additional years of data would be necessary to definitely conclude, one can expect that the seasonal variations are less apparent at Maidanak than at Paranal. The average $200 \mathrm{mB}$ wind velocity above Paranal over a time span of 12 years is $31.3 \mathrm{~m} / \mathrm{s}$, only $12 \%$ more than the 1991 average of Maidanak, but the maxima reached at Paranal during southern winter months are $50 \%$ larger than any of the Maidanak monthly records.
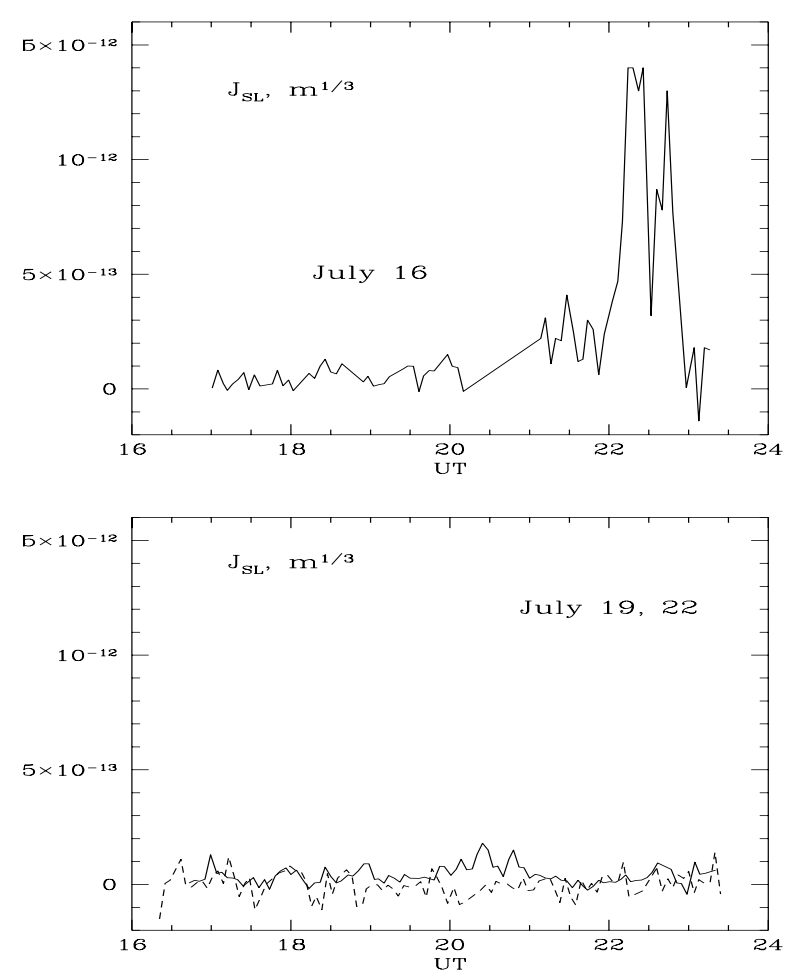

Fig. 17. The evolution of the surface layer turbulence on three nights. The integral of $C_{\mathrm{n}}^{2}$ from 1.9 to $3.4 \mathrm{~m}, J_{\mathrm{SL}}$, is plotted. On July 16 (top) very strong surface layer turbulence was present in the second half of the night. On the nights of July 19 and 22 with good seeing (bottom, full and dashed lines respectively), little turbulence in the surface layer was detected

\section{Relative contribution of the different layers to the seeing at Maidanak}

\subsection{Surface layer}

During night time the air near ground is subjected to a very strong radiative cooling because of the low humidity (Maidanak is located in a semi desert area). The high positive temperature gradient causes large temperature fluctuations in the first few meters above the ground.

Micro-thermal measurements in the surface layer at Maidanak were performed over several years (see summary in Gur92). The altitude dependence of $C_{\mathrm{T}}^{2}$ was found to be approximately as $h^{-2}$. However, the overall contribution of the surface layer to the total seeing degradation was found to be small.

As explained in Sect. 3, the configuration of the GSM at Mt. Maidanak when module 4 was installed at a lower height of $1.9 \mathrm{~m}$ and modules $1-3$ at $3.4 \mathrm{~m}$ allowed us to measure directly the effect of the surface layer. The turbulence integral in the surface layer $J_{\mathrm{SL}}$,

$J_{\mathrm{SL}}=\int_{1.9 \mathrm{~m}}^{3.4 \mathrm{~m}} C_{\mathrm{n}}^{2}(h) \mathrm{d} h$, 
was estimated from the difference of seeing measured by identical modules 3 and 4 with a typical precision of $110^{-13} \mathrm{~m}^{1 / 3}$. The relative contribution of the surface layer to the total $C_{\mathrm{n}}^{2}$ integral was typically less than $20 \%$.

Using the $C_{\mathrm{T}}^{2} \propto h^{-2}$ model of Gur92, we have estimated the difference between seeing measured by DIMM and GSM due to their different heights above the ground. If $1.9-3.4 \mathrm{~m}$ layer contributes about $20 \%$ to the total seeing, then a $3.4-6 \mathrm{~m}$ layer contribution should be $10 \%$. Hence, the DIMM-GSM systematic difference must be $0.9^{3 / 5}=0.94$. It is exactly the ratio of the median seeing measured by both instruments (see Sect. 3.5).

However, at a site with good seeing like Maidanak, in some special circumstances, the local turbulence may become important. Depending on wind direction and velocity, surface layer may significantly contribute to seeing degradation, especially in a location like DIMM's which is not on the very top of the mountain, or in the vicinity of buildings. Local seeing effects were also observed at Paranal (Martin et al. 2000).

The most dramatic surface layer effect was found on July 16, 1998 (Fig. 17, top). Surface layer alone caused seeing degradation up to $1.4^{\prime \prime}$. This corresponded to the period of the worst seeing measured throughout this campaign. A more typical example of two nights with good seeing is shown in the lower plot of Fig. 17. Usually the surface layer contribution is barely apparent, its net effect being on the level of $110^{-13} \mathrm{~m}^{1 / 3}\left(0.3^{\prime \prime}\right.$ seeing) or less.

\subsection{Boundary layer}

The joint contribution of surface and boundary layers (up to $0.5 \mathrm{~km}$ ) to the total $C_{\mathrm{n}}^{2}$ integral was estimated in Gur92 to be typically $12 \%$. Now we know that in that paper the total $C_{\mathrm{n}}^{2}$ integral was overestimated by a factor of $1.4-1.6$, because of the bias in the optical seeing monitor used. This factor follows from comparison of our seeing data with the previous data (Ilyasov et al. 1999) and was already suspected in Gur92. It means that the boundary layer contribution must be increased to about $20 \%$.

\subsection{Free atmosphere}

The $C_{\mathrm{n}}^{2}$ integral in the free atmosphere (roughly above $2 \mathrm{~km}$ ) have been recently estimated in (Kornilov et al. 2000; Kornilov \& Tokovinin 2000) from the measurements of stellar scintillation with apertures of different diameters. These measurements were taken in 1998-99 on a total of 42 nights. The median free atmosphere seeing was estimated as $0.39^{\prime \prime}$. Comparing it to a median seeing of $0.69^{\prime \prime}$, free atmosphere contribution is about $(0.39 / 0.69)^{5 / 3}=0.39$. It was found that the degradation of the free atmosphere seeing was related to the appearance of strong turbulent layers at altitudes of $\approx 3 \mathrm{~km}$ above the summit.
Looking at the wind profile (Fig. 15), we remark that above $2.2 \mathrm{~km}$ a wind shear appears, corresponding to the transition between boundary layer and the western planetary circulation in the free atmosphere. The Pamir mountain system to the east of Maidanak, about $6 \mathrm{~km}$ a.s.l. (3.4 $\mathrm{km}$ above summit) must be a factor defining this transition. So, the true boundary layer at Maidanak is up to $3 \mathrm{~km}$, and not up to $0.5 \mathrm{~km}$ as assumed in Gur92. It means that the contribution of a boundary layer is somewhat higher, partly explaining the apparent discrepancy between Gur92 and (Kornilov et al. 2000).

Summarizing all available information, we suggest the following typical contribution of different layers to the total seeing: surface layer together with a boundary layer up to $3 \mathrm{~km}$ above summit provides some $70 \%$, the remaining $30 \%$ being due to the free atmosphere.

These very preliminary considerations show the importance of the turbulence profile measurements for further characterization of Maidanak atmospheric physics. This provides a strong motivation for future site testing campaigns.

\section{Conclusion}

In this paper we have summarized the results of astroclimate studies at Maidanak observatory in Uzbekistan in 1996-1999, as well as some earlier data. The amount of clear night time hours and its distribution over the seasons for Mt. Maidanak is typical of Central Asian mountains. It is about $58 \%$ of available yearly dark time. In absolute units it corresponds to 2000 hours. But unlike Paranal where the seasonal variations of the available clear time are small, the summer observing capacity of Mt. Maidanak is two times higher than in winter (about 90\% in July and up to $50 \%$ in February). These conditions appear very similar to La Silla, where the same $58 \%$ of yearly clear time has a maximum during the southern winter months.

A survey of the main parameter of atmospheric turbulence (seeing) was carried out at Mt. Maidanak for period from August 1996 to November 1999 with the DIMM, also used for site testing at ESO observatories of La Silla and Paranal in Chile. The last point allows us to characterize seeing conditions of Mt. Maidanak with an instrument calibrated at ESO observatories (Pedersen et al. 1988). From the whole data span, the mean value of seeing is $0.76^{\prime \prime}$, the median is $0.69^{\prime \prime}$. The best monthly median seeing, $0.62^{\prime \prime}$, was observed in November. A median seeing at Mt. Maidanak appears to be better than at La Silla and as good as at Paranal and Roque de los Muchachos observatories.

During the whole period of seeing measurements the temperature, wind velocity and direction were recorded on top of the $5 \mathrm{~m}$ high DIMM tower. A weak correlation of seeing with wind speed is found: the best seeing occurs with light wind up to $5 \mathrm{~m} / \mathrm{s}$. 
In July 1998, a site testing campaign for the estimation of atmospheric parameters related to high angular resolution astronomical techniques was organized at Mt. Maidanak, by the common efforts of University of Nice, ESO, Sternberg Astronomical Institute and UBAI and under INTAS funding. During 9 nights the median wavefront outer scale $\mathcal{L}_{0}=25.9 \mathrm{~m}$ and the median isoplanatic angle $\theta_{0}=2.48^{\prime \prime}$ were measured with GSM. The corresponding median values of those parameters for La Silla, $25 \mathrm{~m}$ and 1.26" (Martin et al. 1998), Paranal, $22.0 \mathrm{~m}$ and 1.90" (Martin et al. 2000) and Cerro Pachon, $36 \mathrm{~m}$ and $2.8^{\prime \prime}$ (Vernin et al. 2000), confirm the suitability of Mt. Maidanak for high resolution observations. The median isoplanatic angle, presented in this paper, is in good agreement with the value of $2.30^{\prime \prime}$ obtained by (Kornilov et al. 2000) from stellar scintillation measurements at Mt. Maidanak in 1998 and 1999, and demonstrates the weak turbulence in the free atmosphere above Maidanak.

A specific feature of the atmosphere above Mt. Maidanak is a very low speed of wavefront motion. The wavefront coherence time $\tau_{0}$, estimated from GSM measurements, appears to be $\approx 12 \mathrm{~ms}$, much larger than for Chilean observatories (Sarazin 1995; Vernin et al. 2000).

The main contribution to the seeing at Mt. Maidanak is produced by the thick boundary layer. The surface layer is not important. On rare occasions when wind blows from the north, and due to the specific location of DIMM $(13 \mathrm{~m}$ lower than the main $1.5 \mathrm{~m}$ telescope), the influence of the surface layer becomes noticeable.

Acknowledgements. The research presented in this paper was supported in part by INTAS-96-367 grant and NATO grant PST.CLG 975586. We are grateful to R. Sunyaev for his efforts in establishing efficient cooperation between ESO and UBAI on Maidanak site testing. We would like also to thank A. Aliev, K. Abdiev, T. Sadibekova, H. Sultanov for participation in the observations and technical assistance, then UBAI administrators A.U. Aliev and F. Uzakov for taking care of the instruments customs clearance and making things run smoothly. The important contributions of prof. F. Martin and R. Conan in GSM mission preparation and data reduction are acknowledged. D. Currie has kindly revised the English of this paper.

\section{References}

Artamonov B.P., Novikov S.B., Ovchinnikov A.A., 1987, in: Methods for increasing the efficiency of optical telescopes, Gladyshev S.A. (ed.). Moscow: Moscow State Univ., p. 16

Avila R., Ziad A., Martin M., et al., 1997, J. Opt. Soc. Am. (A) A14, 3070
Conan R., Ziad A., Tokovinin A., et al., 1999, ASP Conf. Ser. 174, 27

Gladyshev S.A., Shirokova M.G., 1987, in: Methods for increasing the efficiency of optical telescopes, Gladyshev S.A. (ed.). Moscow: Moscow State Univ., p. 45

Gur'yanov A.E., Kalistratova M.A., Kutyrev A.S., et al., 1992, A\&A 262, 373 (Gur92)

Herbst W., 1999, BAAS 31, 646

Ilyasov S.P., Kutyrev A.S., Tokovinin A.A., et al., 1992, Preprint SAI 21, 3

Ilyasov S.P., Baizhumanov A.K., Sarazin M., et al., 1999, Astron. Lett. 25, 122

Kardopolov V.I., Slutsky V.E., Khetselius V.G., Shevcheko V.S., 1971, Astron. Tsirk. 643, 2

Kardopolov V.I., Philip'ev G.K., 1979, Pis'ma v Astron Zh. 5, 106

Kornilov V., Tokovinin A., Vozyakova O., et al., 2000, INTAS 96-367 Final Report, Part III

Kornilov V., Tokovinin A., 2000, Astron. Reports (submitted)

Martin F., Tokovinin A.A., Agabi A., et al., 1994, A\&AS 108, 173

Martin F., Tokovinin A., Ziad A., et al., 1998, A\&A 336, L49

Martin F., Conan R., Tokovinin A., et al., 2000, A\&AS (accepted)

Muñoz-Tuñon C., Vernin J., Varela A.M., 1999, A\&AS 125, 183

Nelsen H.E., 1999, http://www.maidanak.org

Novikova G.V., 1970, in: Atmospheric Optics, Divari N.B. (ed.). Moscow: Nauka, p. 10

Pedersen H., Rigaut F., Sarazin M., 1988, The Messenger 53, 8

Roddier F., 1981, Wolf E. (ed.), Progr. Opt. 19, 281

Sarazin M., Roddier F., 1990, A\&A 227, 294

Sarazin M., 1995, ESO-OSA Top. Meeting on Adaptive Optics, Garching, October 2-6, 439

Sarazin M., 1997a, Proc. SPIE 3125, 366

Sarazin M., 1997b, The Messenger 90, 5

Sarazin M., 2000, http://www.eso.org/gen-fac/pubs/astclim

Schulte-Ladbeck R.E., 1997, BAAS 29, 537

Scott W.B., 1995, Av. Week Space Tech. 15 Nov., 68

Shcheglov P.V., Gur'yanov A.E., 1991, Soviet Astron. 68, 632

Shevchenko V.S., 1973, Soviet Astron. 50, 632

Slutsky V.E., Khetselius V.G., 1970, Astron. Tsirk. 573, 1

Tokovinin A.A., 1980, Soviet Astron. Lett. 6, 386

Tokovinin A.A., Ziad A., Martin F., et al., 1998, Proc. SPIE 3353, 1155

Vernin J., Agabi A., Avila R., et al., 2000, Gemini Report AO-G0094

Wilson R.W., 2000, http://www.ing.iac.es/hap/dimm.html

Zheleznyakova A.I., 1984, in: Astroclimate and efficiency of telescopes, Shevchenko V.S., Kurmaeva A.H. (eds.). Moscow: Nauka, p. 55

Ziad A., Conan R., Tokovinin A., et al., 2000, Appl. Opt. (submitted) 